\title{
Simplifying Autonomic Enterprise Java Bean Applications via Model-driven De- velopment: a Case Study
}

\author{
Jules White, Douglas C. Schmidt, ANIRUdDHA GoKhale \\ Vanderbilt University, Department of Electrical Engineering and Com- \\ puter Science, Box 1679 Station B, Nashville, TN, 37235 \\ \{jules, schmidt, gokhale\}@dre.vanderbilt.edu \\ (251)-533-9432
}

\begin{abstract}
Autonomic computing systems aim to reduce the configuration, operational, and maintenance costs of distributed applications by enabling them to self-manage, selfheal, and self-optimize. This paper provides two contributions to the Model-Driven Development (MDD) of autonomic computing systems using Enterprise Java Beans (EJBs). First, we describe the structure and functionality of an MDD tool that visually captures the design of EJB applications, their quality of service (QoS) requirements, and the autonomic properties applied to their EJBs. Second, the paper describes how MDD tools can generate code to plug EJBs into a Java component framework that provides autonomic capabilities.
\end{abstract}

Keywords: Autonomic Applications Model-Driven Development Enterprise Java Beans

\section{Introduction}

Autonomic computing challenges. Developing and maintaining enterprise applications is hard, due in part to their complexity and the 
impact of human operator error, which have been shown to be a significant contributor to distributed system repair and down time [19]. The aim of autonomic computing is to create distributed applications that have the ability to self-manage, self-heal, self-optimize, self-configure, and self-protect [13], thereby reducing human interaction with the system to minimize down-time from operator error. Although the benefits of autonomic computing are significant [13], the pressures of limited development timeframes and inherent/accidental complexities of largescale software development have discouraged the integration of sophisticated autonomic computing functionality into distributed applications. Some enterprise application platforms, such as Enterprise Java Beans (EJB) [17], offer limited autonomic features, such as application server clustering capabilities, though they tend to have large development teams and long development cycles.

A key challenge limiting the use of autonomic features in enterprise applications today is the lack of design tools and frameworks that can (1) alleviate the complexities stemming from the use of ad hoc methods and (2) generate code that mirrors the specifications of the model. Some infrastructure does exist, such as IBM’s Autonomic Computing Toolkit [10], which focuses on system-level logging and management. System-level autonomic toolkits are inadequate, however, for finegrained autonomic capabilities, such as adjusting algorithms to handle different request demands, which are intended to fix problems early before an entire application must be restarted.

To address the limitations with system-level autonomic toolkits, component-level autonomic frameworks are needed to reduce the effort of 
developing autonomic applications. Component-level autonomic properties support more fine-grained healing, optimization, configuration, monitoring, and protection than system-level toolkits. For example, a mission-critical command and control system for emergency responders should be able to shutdown/restart application components selectively as they fail, rather than shutdown/restart the entire application. With existing autonomic infrastructure based on the system-level, the failure of a key component triggers a restart of the entire application [4], which can incur excessive overhead, particularly for Java-based systems due to JVM initialization latency. In contrast, a componentlevel autonomic framework provides mechanisms to restart only the point of failure [3].

Creating applications with either system or component-level autonomic frameworks requires moving large amounts of state data, analysis data, actions plans, and execution commands between components. These types of applications also require careful weaving of monitoring, analysis, planning, and execution logic into the functional components of the system. Analyzing the autonomic aspects of the application manually, such as checking whether the right state is being monitored by the right components, is a complex process.

Simplifying autonomic system development via MDD techniques. Model-driven development (MDD) [21] is a generative software paradigm that combines

- Domain-Specific Modeling Languages (DSMLs) whose type systems formalize the application structure, behavior, and requirements within particular domains, such as software defined radios, avionics mission 
computing, online financial services, warehouse and freight management, or even the domain of middleware platforms. DSMLs are described using metamodels, which define the relationships among concepts in a domain and precisely specify the key semantics and constraints associated with these domain concepts. Developers use DSMLs to build applications using elements of the type system captured by metamodels and express design intent declaratively rather than imperatively.

- Transformation engines and generators that analyze certain aspects of models and then synthesize various types of artifacts, such as source code, simulation inputs, XML deployment descriptions, or alternative model representations. The ability to synthesize artifacts from models helps ensure the consistency between application implementations and analysis information associated with functional and QoS requirements captured by models. This automated transformation process is often referred to as "correct-by-construction," as opposed to conventional handcrafted "construct-by-correction" software development processes that are tedious and error-prone.

MDD tools are a promising means of reducing the cost associated with creating and validating autonomic computing systems. Models of autonomic systems developed with MDD tools can be constructed and checked for correctness (semi-)automatically to ensure that application designs meet autonomic requirements. Tools can also generate the various capabilities to move data, coordinate actions, and perform other autonomic functions. 
To address the need for component-level autonomic computing - and to avoid ad hoc techniques that manually imbue autonomic qualities into distributed applications - we have created the J3 Toolsuite, which is an open-source MDD environment that supports the design and implementation of autonomic applications. J3 consists of several MDD tools and autonomic computing frameworks, including (1) J2EEML, which captures the design of EJB applications, their quality of service (QoS) [21] requirements, and the autonomic adaptation strategies of their EJBs via a domain-specific modeling language (DSML) [14], (2) Jadapt, which is a J2EEML model interpreter that analyzes the QoS and autonomic properties of J2EEML models, and (3) JFense, which is an autonomic framework for monitoring, configuring, and resetting individual EJBs [6].

This paper describes the structure and functionality of J2EEML and shows how it simplifies autonomic system development by providing notations and abstractions that are aligned with autonomic computing, QoS, and EJB terminology, rather than low-level features of operating systems, infrastructure middleware platforms, and third-generation programming languages. We also describe how (1) Jadapt generates EJB and Java code from J2EEML models to ensure that autonomic applications meet their specifications and to reduce implementation time and (2) JFense provides a set of reusable autonomic components that allow developers to plug-in EJB applications and focus on autonomic logic, rather than the glue for constructing autonomic systems. Finally, we present a case study that qualitatively and quantitatively evaluates how 
the J3 Toolsuite reduces the complexity of developing an autonomic EJB application.

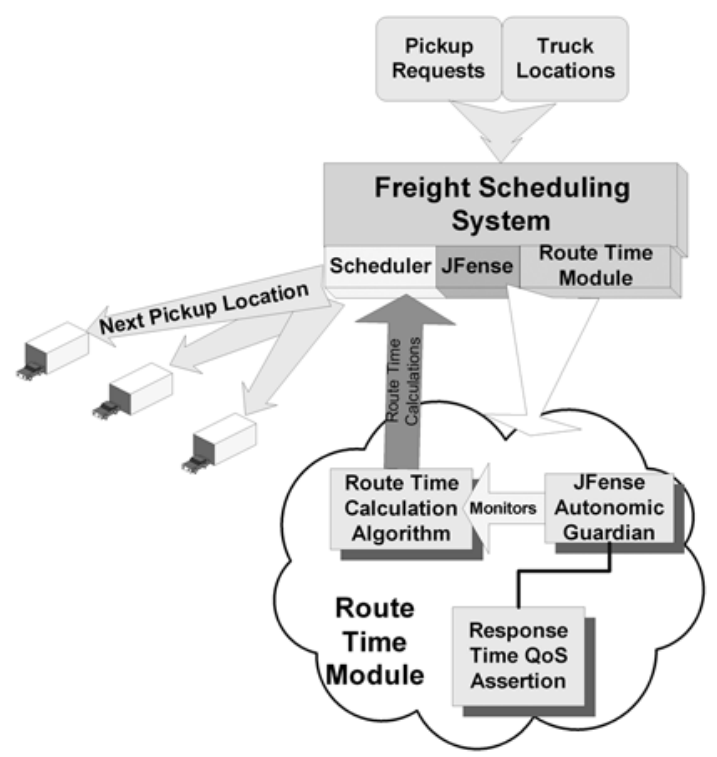

Fig. 1. An Autonomic Architecture for Scheduling Highway Freight Shipments

Our case study centers on an EJB-based Constraint Optimization aNd Scheduling sysTem (CONST) that schedules highway freight shipments using the multi-layered autonomic architecture shown in Figure 1. The system has a list of freight shipments that it must schedule. It uses a constraint-optimization engine to find a cost effective assignment of drivers and trucks to shipments.

A central component in Figure 1 is the Route Time Module (RTM), which determines the route time from a truck's current location to a shipment start or end point. The RTM uses a geo-database and the GPS coordinates from the truck to perform the calculation. This module is critical to the proper operation of the optimization engine. Since a 
heavy load is placed on the $R T M$, it must be designed to maintain its QoS assertions, such as ensuring that the RTM does not exceed a maximum response time of 100 milliseconds. QoS assertions are properties that the system can introspectively measure about itself to determine whether the measured value for the property is beneficial to the system. These measured QoS goals allow the system to decide whether it is in a good state and predict whether it will continue to remain in a good state.

Paper organization. The remainder of this paper is organized as follows: Section 2 describes the MDD J3 Toolsuite for developing autonomic EJB applications; Section 3 gives an overview of J2EEML and describes key challenges we faced when developing it; Section 4 quantifies the reduction in manual effort achieved by using the J3 Toolsuite on CONST; Section 5 compares our work with related research; and Section 6 presents concluding remarks.

\section{The J3 Toolsuite for Autonomic System De- velopment}

The J3 Toolsuite contains the following MDD tools and component middleware frameworks that address the challenges of developing autonomic EJB applications:

- J2EEML, which is a DSML-based MDD tool tailored for designing autonomic EJB applications. J2EEML uses visual representations to model domain-specific abstractions, such as beans, QoS 
properties, and adaptations. J2EEML also provides an automated mapping from QoS requirements to application components.

- Jadapt, which is an MDD tool that produces many artifacts required to implement autonomic EJB applications modeled in J2EEML. Jadapt generates code that meets the J2EEML specifications and also reduces the amount of code that application developers must write manually.

- JFense, which is an autonomic framework that provides components for monitoring, analysis, planning, and execution. Developers can use these components to avoid writing custom autonomic frameworks. JFense can be configured to meet the autonomic requirements for a range of EJB applications.

This section focuses on the design and function of J2EEML and illustrates how it can be used to create structural models of EJB applications.

J2EEML is a DSML that enables EJB developers to construct models that incorporate autonomic and QoS concepts as first-class entities. J2EEML itself was developed for both the Generic Modeling Environment (GME) [15] and the Generic Eclipse Modeling System (GEMS) [22], which are general-purpose MDD environments that we use to simplify the creation of metamodels and model interpreters. Metamodels characterize the roles and relationships in the autonomic computing domain, and model interpreters generate many artifacts required to implement autonomic EJB applications. J2EEML captures the relationship between QoS assertions and application components to address key design challenges of developing autonomic applications. 
For example, J2EEML helps developers understand which components to monitor in their EJB applications by enabling them to visualize and analyze the relationships between components and QoS assertions.

Developers use J2EEML to capture the design of autonomic systems and the mapping of components to QoS assertions in four phases: (1) they create a structural model of the EJBs comprising an autonomic system, (2) they create models of the QoS properties that the system is attempting to maintain, (3) they map these QoS properties to the specific beans within the system that the properties are measured from, and (4) they design courses of action to take when the desired QoS properties are not maintained. This modeling process captures the structure of the system, how the QoS properties are related to the structure, and what adaptation should occur if a QoS property is not within an acceptable range.

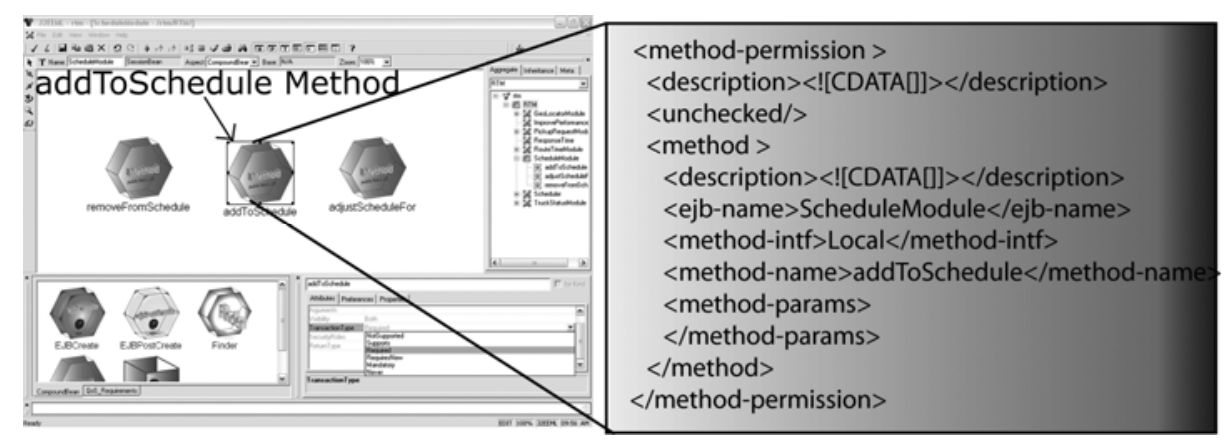

Fig. 2. J2EEML Remote Interface Composition Model for the TruckStatusModule

\subsection{Modeling EJB Structures with J2EEML}

The first piece of a J2EEML model is its EJB structural model, which describes the components of the system that will be managed 
autonomically. This model defines the beans that compose the system and captures the EJB specifics of each bean, including JNDI names, transactional requirements, security requirements, package names, descriptions, remote and local interface composition, and bean-to-bean interactions. An EJB structural model is constructed via the following six steps:

1. Each session bean is represented by dragging and dropping session bean atoms into the J2EEML model. Developers then provide the Java Naming and Directory Interface (JNDI) name of each bean, its description, and its state type (i.e., stateful or stateless).

2. For each session bean, a model is constructed of the business methods and creators supported by the bean by dragging and dropping method and creator atoms. Figure 2 shows a model of the remote interface composition of the TruckStatusModule from CONST.

3. Entity beans are dragged and dropped into the model to construct the data access layer. These beans are provided a JNDI name/description and properties indicating if they use container managed persistence (CMP) or bean managed persistence (BMP).

4. Persistent fields, methods, and finders are dragged and dropped into the entity beans. Each persistent field has properties for setting visibility, type, whether it is part of the primary key, and its access type (i.e., read-only or read-write).

5. Relationship roles are dragged and dropped into the entity beans and connected to persistent fields. These relationship roles can be connected to other relationship roles to indicate entity bean relationships. 
6. Connections are made between beans to indicate bean-to-bean interactions. Capturing these interactions allows Jadapt to later generate the required JNDI lookup code for a bean to obtain a reference to another bean.

After these six steps have been completed, the J2EEML model contains enough information to represent the composition of the EJBs.
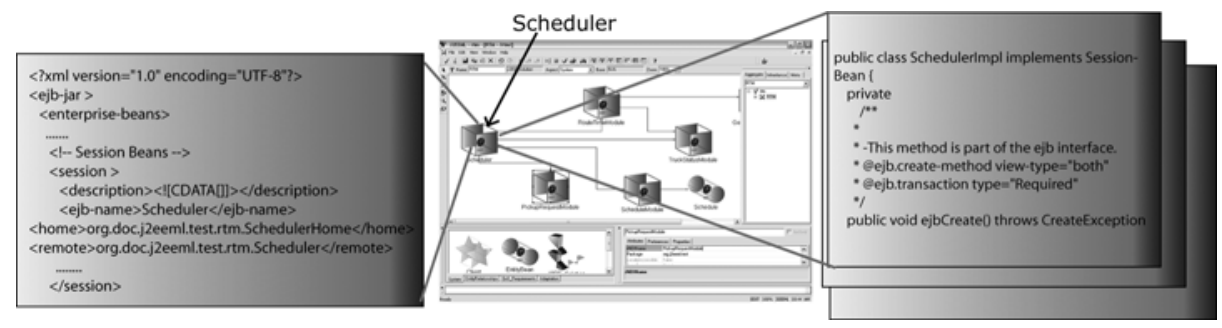

Fig. 3. J2EEML Structural Model Showing Bean-to-Bean Interactions

Figure 3 shows a J2EEML structural model of CONST. In this figure, each bean within CONST has been modeled via J2EEML. Interactions between the beans are also modeled, thereby allowing developers to understand which beans interact with one another. Figure 3 also illustrates snippets of the XML deployment descriptor and Java class generated for the Scheduler.

To support decomposition of complex enterprise architectures into smaller pieces, J2EEML allows EJB structural models to contain child EJB structural models or subsystems. Beans within these children show up as ports that can receive connections from the parent solution. This hierarchical design allows developers to decompose models into manageable pieces and enables different developers to encapsulate their designs. 
For CONST, we constructed a structural model of each bean required for the Route Time Module, constraint-optimization engine, truck status system, and incoming pickup request system, as shown in Figure 3. The model also includes information on the entity beans used to access the truck location and pickup request databases.

Using J2EEML provides several advantages in the design phase, including (1) visualization of beans and their interactions, component security requirements, system transactional requirements, and interactions between beans, (2) enforcement of EJB best practices, such as the Session Façade pattern [1], which hides Entity beans from clients through Session beans, and (3) model validation, including checks for proper JNDI naming. J2EEML's visualization benefits significantly decrease the difficulty of understanding system structure and interactions. The model validation and enforcement of best design practices facilitate rapid creation of a well-designed solution that is correctby-construction.

\section{Designing J2EEML to Address Key Con- cerns of Autonomic Computing}

Autonomic applications require four elements to achieve their goals: monitoring, analysis, planning, and execution [13]. These elements form a controller that observes and adapts the application to maintain its QoS goals, such as maintaining a minimum response time of $100 \mathrm{~ms}$ for requests. This section describes how the monitoring, analysis, and planning aspects of autonomic systems presented unique challenges 
when designing and building J2EEML and shows how we addressed each challenge. To focus the discussion, we use the Route Time Module $(R T M)$ shown in Figure 1 as a case study to illustrate key design challenges associated with autonomic systems.

\subsection{Monitoring}

Monitoring is the phase in autonomic systems where applications observe their own state. Since this state information is used in later phases to control system behaviors it is crucial that the right information be collected at the right times without adversely impacting system functionality and QoS. The following are key design challenges faced when developing the monitoring aspects of autonomic systems:

Challenge 3.1.1: Providing the ability to specify the large range of data that can be monitored by the system. Developers of autonomic systems must address the issue of how to self-monitor key data, e.g., by capturing CPU and memory utilization, exceptions thrown by the application, or error messages in a log. The model for specifying what information to capture from the system must be flexible and support a range of data types. The model must also be extensible and support unforeseen future data types that might be needed later.

A core concept behind J2EEML is that an autonomic EJB application can measure properties of its current state introspectively and determine if the property values indicate the application is in a safe or optimal state. J2EEML models the properties it measures via QoS assertions, which determine which properties an autonomic system can introspectively measure and analyze to determine if the properties are in 
an acceptable assertion range. Each assertion provides properties for setting its name and description. Developers can drag and drop these assertions into J2EEML models.

The J2EEML QoS assertions model is critical for understanding an autonomic system's QoS properties, how they can be measured, what their values should be, and how degradations in them can be corrected. Understanding QoS assertions is also crucial to designing the structural architecture of EJB applications and understanding how they meet those assertions. Capturing and mapping QoS requirements to the appropriate structural architecture have traditionally used natural language descriptions, such as "the service must support 1,000 simultaneous users with a good response time.” Due to the lack of an unambiguous formal notation, such descriptions are prone to different interpretations, which result in architectures that do not meet the QoS requirements. Choosing an EJB architecture that best fits the QoS requirements can be complex and error-prone since specification ambiguity and hidden architectural trade-offs make it hard to choose the appropriate design.

For example, deciding whether to use remote interfaces for a J2EE implementation of a service can have a substantial impact on end-toend system QoS. Remote interfaces allow distribution of beans across servers, which can increase scalability and reliability. Distribution can also increase latency, however, since requests must travel across a network or virtual machine boundaries.

With the RTM in our case study, one QoS assertion is the average response time. This QoS assertion states that the system will measure all 
requests to the $R T M$ and track the average time required to service each request. If the calculated average response time exceeds 50 milliseconds, the assertion is false, indicating that the RTM is taking too long to respond, otherwise the assertion is true, indicating that the RTM is responding properly.

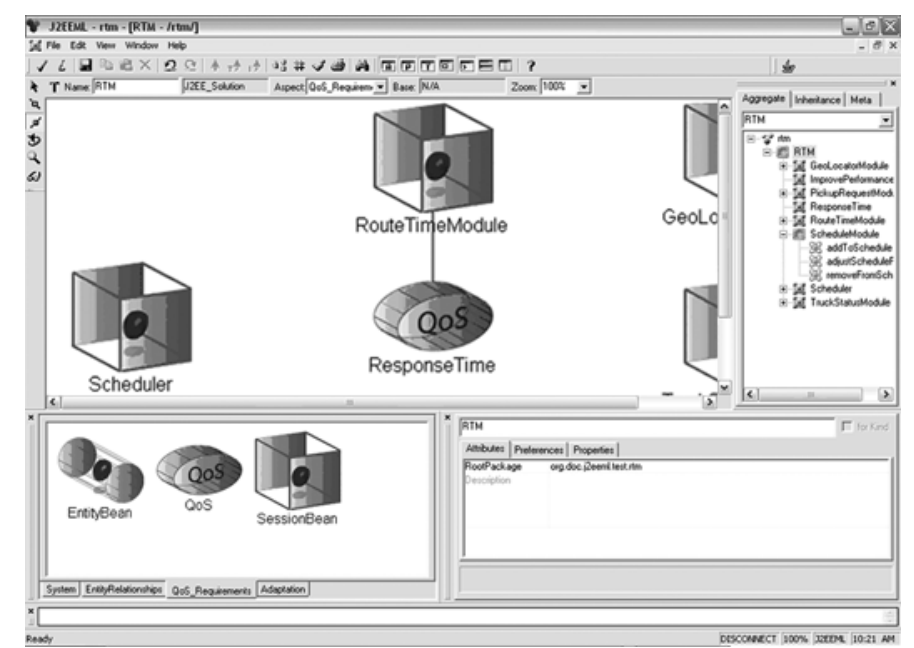

Fig. 4. J2EEML Model Associating the ResponseTime QoS Assertion with the RouteTimeModule

Figure 4 illustrates a J2EEML model of the scheduling system and the association of the RTM to the ResponseTime QoS property. This model shows J2EEML's ability to model QoS properties as aspects [16] that are applied to a component. When the model is interpreted and the Java implementation generated, the association between the $R T M$ and $R e$ sponseTime assertion will generate the appropriate monitoring code in the RTM's implementing class.

Challenge 3.1.2: Building a system to specify where monitoring logic should reside in the system. The decision of what to monitor directly affects where the monitoring logic will reside. To monitor a log 
for errors, the logic could be at any level of the application, such as a central control level. For observing exceptions or the load on a specific subcomponent of the application, the monitoring logic must be embedded more deeply. In particular, developers must position the monitoring capability precisely so that it is close enough to capture the needed information, but not so deeply entangled in the application logic that it adversely affects performance and separation of concerns [20].

In CONST, for example, we must ensure separation of concerns in the application design and find an efficient means of monitoring. A natural approach to collecting request statistics for the RTM might be to simply add the appropriate state collection code into the route time logic. The monitoring logic for the RTM, however, should not be entangled with the route time calculation logic and reduce its readability and maintainability. Moreover, the time to monitor and analyze each request should be insignificant compared to the time to fulfill each route request.

After the structural and QoS assertion models are completed, developers can use J2EEML to map QoS assertions to EJBs in the structural model. This mapping documents which QoS assertions should be applied to each component. It also indicates where monitoring, analysis, and adaptation should occur for an autonomic system to maintain those assertions. For example, to determine the average response time of the $R T M$, calls to the RTM's route time calculation method must be intercepted to calculate their servicing time. The relationship between the $R T M$ bean and average response time assertion in the model indicates 
that the RTM bean must be able to monitor its route time calculation requests.

J2EEML supports aspect-oriented modeling [8] of QoS assertions, i.e., each QoS assertion in J2EEML that crosscuts component boundaries can be associated with multiple EJBs. For example, maintaining a maximum response time of 100 milliseconds is crucial for both the $R T M$ and the Scheduler bean. Connecting multiple components to a QoS assertion, rather than creating a copy for each EJB, produces clearer models. It also shows the connections between components that share common QoS assertions. Figure 5 shows a mapping from QoS assertions to EJBs. Both the RTM and the Scheduler in this figure are associated with the QoS assertions ResponseTime and AlwaysAvailable. The ResourceTracker and ShipmentSchedule components also share the AlwaysAvailable QoS assertion in the model.

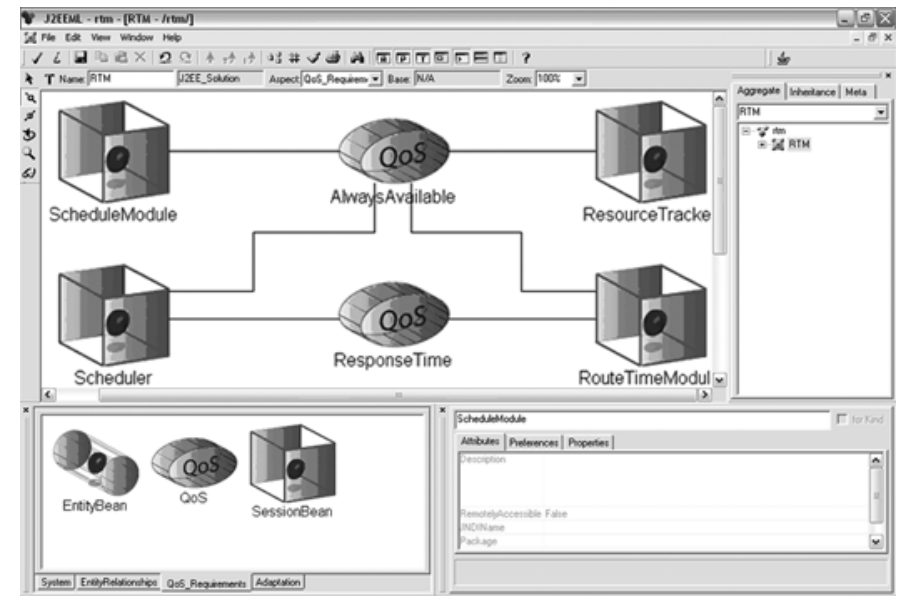

Fig. 5. J2EEML Mapping of QoS Assertions to EJBs

Components can have multiple QoS assertion associations, which J2EEML supports by either creating a single assertion for the compo- 
nent that contains sub-assertions or by connecting multiple QoS assertions to the component. If the combination of assertions produces a meaningful abstraction, hierarchical composition is preferred. For example, the RTM is associated with a QoS assertion called AlwaysAvailable constructed from the sub-assertions NoExceptionsThrown and NeverReturnsNull. Combining MinimumResponseTime and NoExceptionsThrown, however, would not produce a meaningful higher-level abstraction, so the multiple connection method is preferred in this case.

\subsection{Analysis}

Analysis is the phase in autonomic systems, which takes state information acquired by monitoring and reasons about whether certain conditions have been met. For example, analysis can determine if an application is maintaining its QoS requirements. The analysis aspects of an autonomic system can be (1) centralized and executed on the entire system state or (2) distributed and concerned with small discrete sets of the state. The following are key challenges faced when developing an autonomic analysis engine:

Challenges 3.2.1: Building a model to facilitate choosing the type of analysis engine and Challenge 3.2.2: Building a model to facilitate choosing how the engine should be decomposed. To choose a hierarchical vs. monolithic analysis engine, the tradeoffs of each must be understood. Concentration of analysis logic into a single monolithic engine enables more complex calculations. However, for simple calculations, such as the average response time of the RTM component, a monolithic engine requires more overhead to store/retrieve state infor- 
mation for individual components than an analysis engine dedicated to a single component. A monolithic analysis engine also provides a central point of failure. A key design question is thus where analysis should be done and at what granularity.

A model to facilitate choosing the appropriate type of analysis engine must enable developers to identify what data types are being analyzed, what beneficial information about the system state can be gleaned from this information, and how that beneficial information can most easily be extracted. It is important that the model enable a standard process for examining the required analyses and determining the appropriate engine type.

To create an effective analysis engine, developers must determine the appropriate hierarchy or number of layers of analysis logic. A key issue to consider is whether an application should have a single-layer vs. a hierarchical multi-layered analysis engine. At each layer, the original monitoring design questions are applicable, i.e., what should be monitored and how should it be monitored? A model to enable these decisions must clearly convey the layers composing the system. It must also capture what analysis takes place at each layer and how each layer of analysis relates with other layers.

In the context of our highway freight scheduling system, a key question is whether the RTM's autonomic layer analyzes its response time or whether a layer above the RTM should do it. At each layer, the analysis design considerations are important too, e.g., what information the system is looking for in the data, how it finds this information, and how this can be better accomplished by splitting the layer. For exam- 
ple, a developer must consider whether every request to the $R T M$ should be monitored to determine if the RTM is meeting its minimum response time QoS. Conversely, perhaps only certain types of requests known to be time consuming should be monitored. Another question facing developers is how the RTM's monitoring logic sends data to its analysis engine.

Developers can use J2EEML to design hierarchical QoS assertions to simplify complex QoS analyses via divide-and-conquer. A hierarchical QoS assertion is only met if all its child assertions are met, i.e., all the child QoS assertions must hold for the parent QoS assertion to hold. With respect to the RTM, the QoS assertion GoodResponseTime only holds if both the child QoS assertions AverageResponseTime and MaximumResponseTime also hold. This hierarchical composition is illustrated in Figure 6, where GoodResponseTime is an aggregation of several properties of the response time.

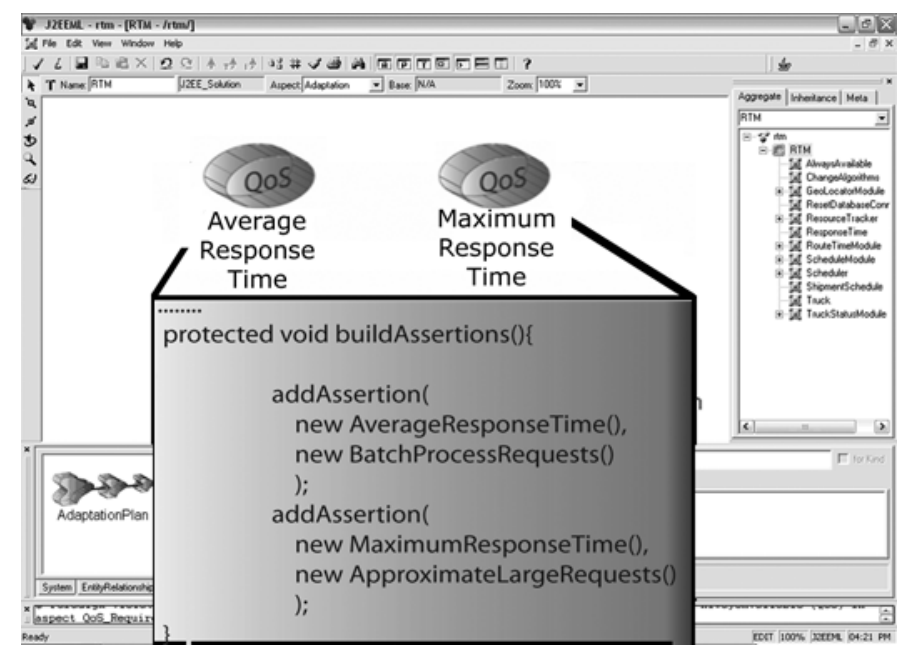

Fig. 6. J2EEML Hierarchical Composition of ResponseTime QoS Assertion J2EEML Hierarchical Composition of ResponseTime QoS Assertion 
Modeling QoS assertions hierarchically can help enhance developer understanding of what type of analysis engine to choose. A small number of complex QoS assertions that cannot be broken into smaller pieces implies the need for a monolithic analysis engine. A large number of assertions - especially hierarchical QoS assertions - implies the need for a multi-layered hierarchical analysis engine.

Modeling QoS assertions hierarchically also enhances developer understanding of how to decompose the analysis engine into layers. The hierarchical model of QoS assertions corresponds directly to the decomposition of the analysis engine into layers. Developers can use J2EEML to first add complex QoS assertions to their models and then determine if the complex assertion can be accomplished by combining the results of several smaller analyses. If so, developers can add these smaller QoS assertions as children of the original QoS assertion to represent the smaller analyses and then apply this iterative process to the new children.

\subsection{Planning}

Planning is the phase in autonomic systems where applications examine the results of their analyses and decide what actions to take to reach their assertions. For our highway freight scheduling example, this could involve changing the RTM to use a less precise but faster algorithm that maintains the minimum response time as demand grows. A typical autonomic application may have hundreds of assertions and planning the correct actions in the face of QoS failures is critical to an autonomic 
application. The following are key challenges faced when developing an autonomic planning engine:

\section{Challenge 3.3.1 Designing a means to specify layered adaptation}

plans. As with monitoring and analysis, planning can be implemented with a layered architecture. A simple, one-layer architecture would monitor, reason, and react to all system events at one level, which works well for macro-level events and actions. For applications that need more flexible and fine-grained control of their behavior this simple one-layer architecture is less suitable. For example, if the RTM needs to switch algorithms in response to a degradation in response time, a small controller located close to the RTM would be able to react more quickly and with less overhead than a larger controller located farther away. If however, the RTM needed to switch algorithms due to a period of high demand predicted from historical data, a small controller located close to the RTM is infeasible since it is unlikely to have access to the appropriate data for the prediction. Moreover, a predicted period of high demand may necessitate changes to components other than just the RTM and thus require a large monolithic controller with access to multiple components. To increase flexibility and fine-grained control, therefore, more layers can be integrated into the system. Layers distribute intelligence throughout the system and support a divide-andconquer approach to planning.

After the planning is provisioned into layers, each layer must be assigned a responsibility to react to and recover from QoS failures. In CONST, one layer ensures that the RTM is always available and the next layer down ensures that a minimum response time is maintained. 
Intelligent separation of responsibilities can produce hierarchical chains of command that reduce the complexity of accomplishing the overall assertion. Finding these well-proportioned divisions of labor is hard.

J2EEML models adaptation by specifying the actions the system should take when a QoS assertion fails. Each application component may have a group of assertions associated with it. If one assertion does not hold for the component, it indicates a QoS failure that must be fixed. Developers can use J2EEML to specify groups of actions that must be taken to correct these failures.

Once an assertion has failed to hold for a specific component, the application must determine how to fix the problem. To model the appropriate course of action, J2EEML uses the concept of adaptation plans, which are groups of actions that can be performed to fix a specific type of QoS assertion failure. For example, if the average response time assertion fails, the RTM must change its calculation algorithms to be less precise but run faster. Figure 7 shows a J2EEML model that associates the ResponseTime QoS assertion with the ChangeAlgorithms single-layered adaptation plan. 


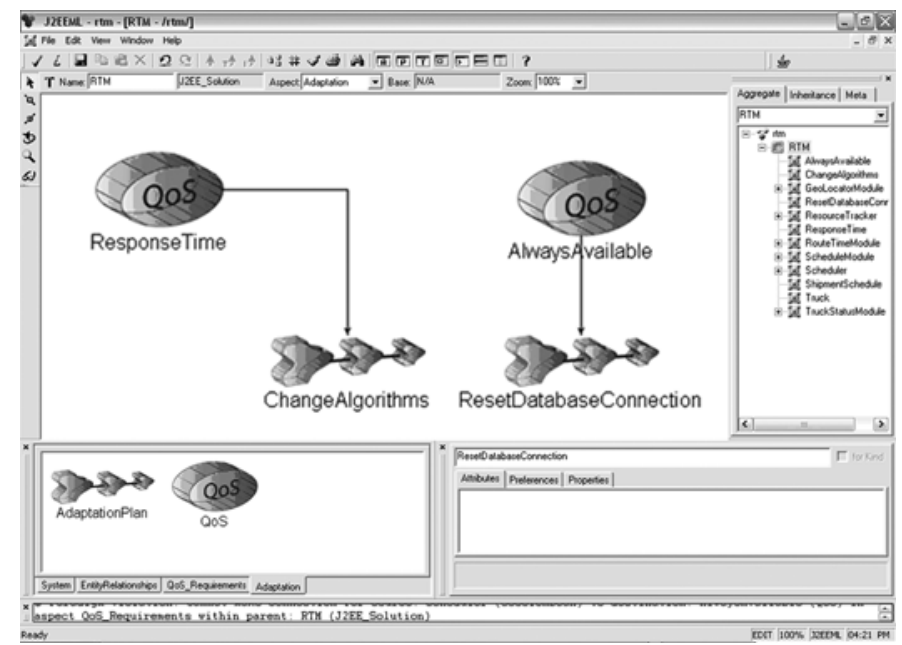

Figure 7: An Association between the ResponseTime QoS Assertion and the ChangeAlgorithms Adaptation Plan

Adaptation plans indicate the responsibilities of an autonomic layer, i.e., the adaptation plan specifies the actions that the autonomic layer can perform in the event of a QoS failure. This association also guides the selection of a single-layer or multi-layered planning architecture. If a complex QoS assertion does not have adaptation plans associated with its children, the proper course of action to take when one of the child QoS assertions fails cannot be determined by the data available to the child. If only top-level QoS assertions have associated adaptation plans, this implies the need for a single planning layer. If, however, the QoS children have adaptation plans associated with them, this implies that they can determine the corrective course of action and require a multi-layered planning solution. 


\subsection{Reducing the Complexity of Developing Auto- nomic Systems with JFense and Jadapt}

JFense is a component-level framework that performs autonomic functions, such as monitoring the QoS of EJBs, analyzing system state, communicating between autonomic layers, determining how to adapt to QoS failures, and executing adaptation plans. Jadapt is a J2EEML model interpreter that supports rapid development and verification of autonomic code by generating implementations of EJBs from a structural model.

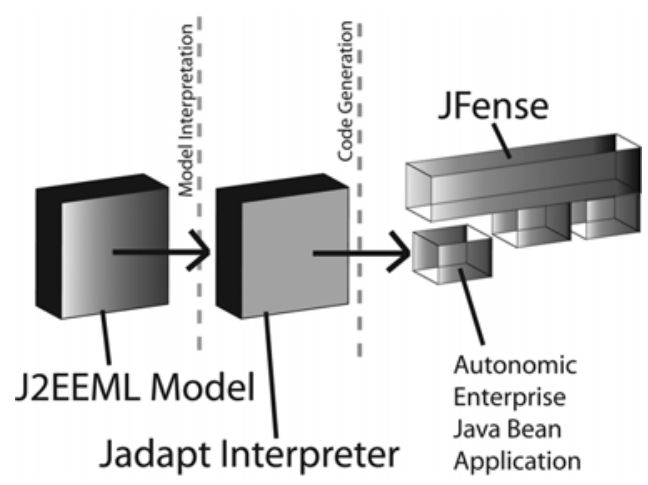

Figure 8, Developing an Autonomic Application with the J3 Toolsuite.

Jadapt serves as a bridge between a J2EEML model and the JFense framework, i.e., it generates Java code for (1) the J2EEML structural model and (2) plugging the generated EJBs into the JFense framework. Jadapt generates configurations for JFense to mirror the J2EEML model, stubs for the EJBs, EJB deployment descriptors, and monitoring, analysis, planning, and execution class stubs, which relieves developers from tedious and error-prone coding tasks. Moreover, Jadapt ensures that the code mirrors the system architecture in the J2EEML 
implementation, which reduces problems stemming from misinterpreting specifications and inconsistencies between interfaces and their implementations.

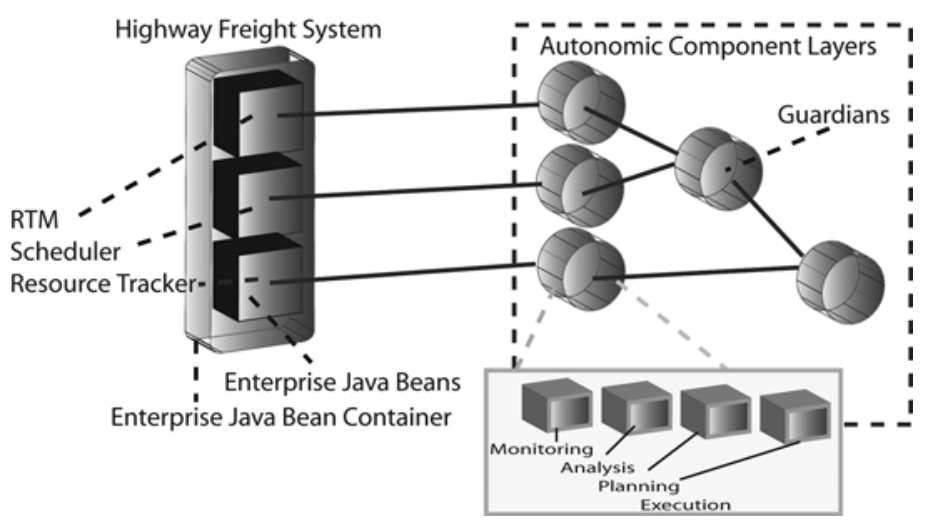

Figure 9, The JFense Architecture

To simplify the development of autonomic EJB applications, we created the JFense framework for constructing autonomic EJB systems.

JFense provides a multi-layered architecture for monitoring, analyzing, planning, and executing in an autonomic system. The basic structure of JFense is defined as follows:

1.Each bean has a guardian class responsible for monitoring its state and running QoS analysis, as shown in Figure 9. The beans push state data out to the guardians using an event-based system. The guardians act as observers on the beans, i.e., they are the key elements for monitoring beans and routing state information to the proper QoS analysis objects.

2.An analysis class for each QoS goal is created. These QoS goals are used by the guardians to analyze the bean's current state and deter- 
mine if it is meeting its QoS requirements. Hierarchical QoS goals are created through aggregation.

3.Each guardian class has an associated action plan for determining the course of action if a QoS goal fails. The guardian also notifies any guardians at the level above when it cannot maintain its QoS goals. When a bean's state changes, it notifies its guardian that a state change event has occurred. The guardian then uses each of its QoS analysis objects to analyze the bean's state and ensure that its objectives are still being met.

Bean requests are the default state information monitored by guardians. Jadapt generates proxies that monitor the input, output, time, and exceptions thrown for each method accessible through the beans local or remote interface and pass it to the Guardians.

Beans monitor requests on their accessible methods through generated proxies. When a request is issued to the bean, the generated proxy first receives the request and notes the starting time. The proxy then notifies the guardian that a request is starting so that any pre-conditions on the request can be analyzed. These pre-conditions can be used to identify QoS failures in other portions of the system, other systems, or clients. The proxy then passes the request to the actual method that contains the logic to fulfill it (we refer to this method as the implementing method). When the implementing method has returned, the bean again notifies its guardian, which enables the guardian to check post-conditions, such as output correctness or servicing time. Finally, the result is passed back to the caller. 
After the state is routed to the analysis object, it determines if its QoS property is being met. JFense has several predefined analysis objects for common functions, such as monitoring request time. Other autonomic analyses can be added by extending the JFense analysis interfaces or implementing the class skeletons generated by Jadapt from the J2EEML model. If the QoS is not being maintained, the analysis object notifies the guardian, which will either directly execute an action plan or propagate the QoS failure event up the chain of guardians.

Guardians also use the Strategy pattern [7] to determine how to react to a QoS failure. Different planning strategies can be plugged into a guardian at design- or run-time to find the appropriate course of action for each QoS failure. Strategies can be plugged in at both design and run-time. The default strategy uses a hashing scheme to associate QoS analysis objects with Command pattern [7] actions, which encapsulate actions as objects, to allow adaptations to be queued, logged, or undone. In the event of a QoS failure, the appropriate action is looked up from the table and executed.

JFense alleviates developers of the need to build an autonomic framework from scratch. In the highway freight scheduling system, for example, JFense handles inter-layer communication so that developers can focus on the logic needed to analyze the state data, determine the correct course of action, and adapt the system. JFense also provides the communication, monitoring, and message bus infrastructure to glue the provided logic together, which significantly reduces the time and effort required to build autonomic applications that monitor their own state and adapt to achieve their goals. 


\section{Evaluating Development Effort Savings of the J3 Toolsuite}

We developed the highway freight scheduling system case study to illustrate the advantages of using the J3 Toolsuite to develop autonomic EJB applications. The initial implementation of this case study required 1200 lines of Java code. The generated EJB implementations accounted for nearly $75 \%$ of the complete code base, the test framework accounted for $20 \%$, and the JFense glue code accounted for $5 \%$. Using a traditional development approach, all of this code would have been developed manually. With the J3 Toolsuite, in contrast, 883 lines of code were generated by Jadapt from our J2EEML specification.

Using our highway freight scheduling case study, we evaluated the impact of adding new sources of information that required monitoring and where the logic would reside. In our initial design, only response times of the Scheduling component were monitored. We then refactored the design to monitor response times of the RTM component, as well. Adjusting the design using J2EEML and re-generating the implementation took approximately five mouse clicks and resulted in the generation of $\sim 20$ new lines of source code that correctly mirrored the specification. This refactoring can be seen in Figure 10. 


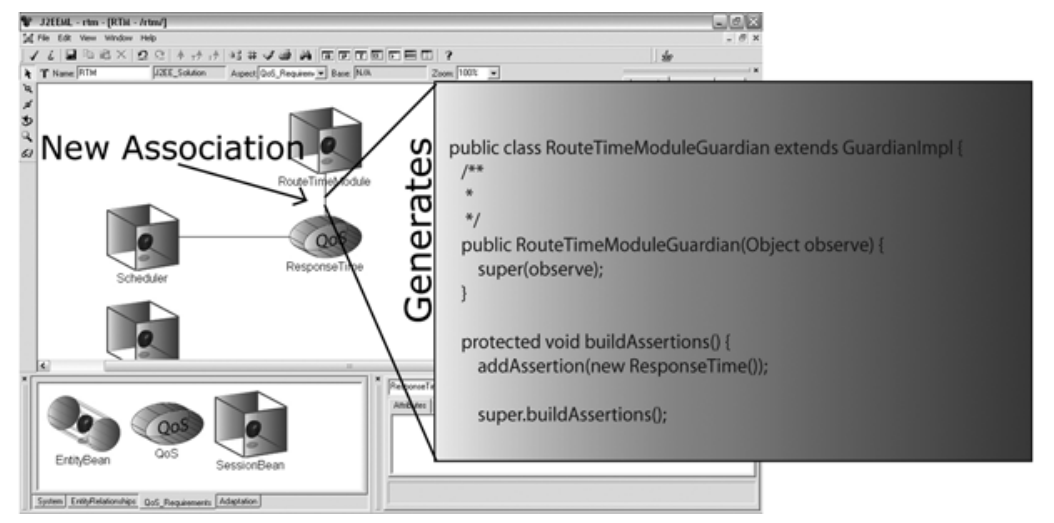

Figure 10, Refactoring the RTM's QoS Assertions

To evaluate the impact of design refactoring on the analysis and planning layers of the highway freight system, we modified its initial design by changing its response time analysis and adaptation into a hierarchy of average and maximum response times. The refactoring in J2EEML was straightforward and took 12 mouse clicks. The change generated $\sim 75$ new lines of code, which minimized the complexity of the design change and implementation update. Again, for large development projects without MDD tool support, many such changes would occur and hence the manual redevelopment effort would be much higher.

To evaluate the development effort associated with sharing adaptation plans between QoS assertions, we refactored our highway freight system to share the improved response time adaptation plan between both the average response time QoS assertion and the maximum response time QoS assertion. After this change was made to the model and Jadapt regenerated the model artifacts, 36 new lines of code were present that updated the existing adaptation plan to include the new adaptations and changed the adaptation plan of the maximum re- 
sponse time to use its modified adaptation plan. As with other refactorings we analyzed, adjusting the J2EEML model and regenerating the code required $\sim 12$ mouse clicks, while developing the equivalent functionality manually required significantly more effort.

As with the autonomic modeling and generation capabilities of the J3 Toolsuite, significant reductions in development complexity were yielded by applying MDD to the implementation of the structural model. For example, when a single SessionBean with one method was added to the J2EEML model, the resulting bean, interfaces, deployment descriptor, and helper classes generated 116 lines of Java code and 80 lines of XML. The model change in J2EEML required two drag and drop operations. As with the autonomic code generated by Jadapt, the code was correct-by-construction and the JNDI name of the bean was also correct. Adding two interactions from existing beans to the new bean generated another 12 lines of error-prone JNDI lookup/narrowing code that was automatically generated by Jadapt, thereby simplifying developer effort and enhancing confidence in the results.

\section{Related Work}

An increasing number of MDD tools exist for modeling componentbased systems. Cadena [9] is an MDD tool for building and modeling component-based DRE systems, with the goal of applying static analysis, model-checking, and lightweight formal methods to enhance these systems. Other tools, such as Rational Rose, provide UML modeling capabilities for component-based systems. In contrast to J2EEML, 
these tools are not tailored to the domain of modeling autonomic functionality in component-based systems. For example, they lack the ability to establish the critical mapping between QoS properties, components, and adaptations, which forces developers to (1) resort to traditional textual descriptions for specifying QoS properties and (2) maintain separate models for understanding how the QoS, adaptation, and components in the system interrelate. As a result, it is hard to understand how an application will monitor itself and how it will react to QoS failures.

Other middleware approaches to managing the QoS of distributed applications are similar to JFense. The Generic Object Platform Infrastructure (GOPI) [5] provides a pluggable and modular platform for the development of middleware. GOPI, in particular, includes support for annotating interface interaction points with QoS attributes. As with J3, there is no limitation on what can be considered a QoS attribute. These attributes are mapped to specific middleware configurations through code to tailor an application's performance. QoS groups can be created to partition the interaction points into sets that share QoS requirements. JFense also provides the ability to associate components that have similar QoS requirements. JFense, however, allows a single component to be associated with multiple QoS groups whereas GOPI does not. In GOPI, each communication protocol can have a QoS manager associated with it to ensure that a communication binding maintains its required QoS. This design is similar to the JFense approach of using Guardian classes to monitor EJBs and notify the appropriate adaptations when QoS degrades. GOPI requires that developers implement 
the planning logic that determines what response should be taken to a QoS degradation. By using the J3 toolsuite, the planning logic is automatically generated from the J2EEML model. Furthermore, adaptations can be written once and incorporated into multiple aspects of an application by merely updating the J2EEML model and regenerating the JFense code. Using a model-driven middleware approach provides significant benefits to the implementation and re-factoring of adaptation logic when compared to hand-coding with a platform such as GOPI. $\mathrm{QuO}$ [23] is another middleware architecture for mapping QoS to objects. In QuO, the state of the operating environment can be partitioned into regions. Transitions between these regions trigger adaptive behavior. This architecture is similar to how JFense operates. With JFense, adaptations occur as assertions become true or false. A key difference between $\mathrm{J} 3$ and Quo is that $\mathrm{J} 3$ is a complete model-driven process for developing adaptive applications and not just a QoS-aware middleware framework. With J3, most of the tedious configuration and implementation code is generated from the modeling tool. As discussed previously, this greatly reduces the cost of re-factoring adaptations as the understanding of the target operating domain improves. Moreover, it decreases the initial entry cost of building an adaptive application.

IBM's Autonomic Toolkit [18] addresses the issues of monitoring, analysis, planning, and executing autonomic applications. It includes the Autonomic Management Engine, which monitors events, analyzes them, then plans and executes corrective action on a computing resource; the Generic Log Adapter [10] for Autonomic Computing, which converts existing log files to the Common Base Event format 
[11]; and the Log and Trace Analyzer for Autonomic Computing, which reads logs in the Common Base Event format, correlates the logs based on different criteria, and displays the correlated log records. These tools do not, however, address the complexity of integrating autonomic functionality into applications, i.e., they do not help developers design their autonomic applications or implementing the logic required by them. In contrast, the $\mathrm{J} 3$ Toolsuite is specifically tailored to reducing design and implementation complexity, as well as providing a runtime framework.

Another related research area is microrebooting [3], which posits that entering unsafe states in large scale systems is unavoidable and can be combated by recursively rebooting larger and larger portions of the system until the unsafe state is cleared. This research is complimentary to the work of J2EEML and JFense. JFense provides a framework whereby rebooting logic can be inserted at the component level to enable microrebooting. Moreover, in J2EEML, application designers can specify exactly which components must support rebooting and use Jadapt to automatically weave the required code into those locations.

\section{Concluding Remarks}

In theory, autonomic systems can minimize the impact of human error in development and management. In practice, however, it is hard to develop the monitoring, analysis, planning, and execution aspects required for autonomic systems reliably and productively since developers must reason about complex sets of QoS assertions and ensure that applications meet them. Model-driven autonomic capabilities pro- 
vide a means for EJB applications to self-manage and attempt to maintain the QoS assertions. To facilitate self-management, the structure of EJB applications and their QoS assertions must be captured in models so applications can reason about themselves.

The bridge between the QoS assertions of autonomic systems and their structural designs involves mapping these assertions to specific system components. Without this mapping, applications cannot use introspection to determine whether their QoS assertions are being met. The J3 Toolsuite described in this paper provides Model-Driven Development (MDD) tools and an autonomic computing framework to support these capabilities to simplify the development of autonomic EJB applications.

The J2EEML MDD tool helps link assertions and structure by allowing developers to specify this mapping via a DSML. J2EEML also includes mechanisms for modeling complex EJB structures, interactions, and architectures and using these models to generate code that mirrors the specifications from the model, which frees developers from reinventing complex autonomic frameworks for each new application.

After capturing structural properties, QoS assertions, and assertion to structure mapping in J2EEML, developers still must integrate autonomic features into their distributed EJB applications. This integration is often complicated due to the lack of component-level frameworks for autonomic systems. To address these concerns, we have developed the Jadapt code generation tool and the JFense autonomic framework. Jadapt allows developers to generate the code needed to plug their application's EJBs into JFense. JFense provides a comprehensive and 
flexible framework for multi-layered autonomic monitoring, analysis, planning, and execution architectures, which allows developers to focus on the system's business logic and QoS analysis logic.

The following are our lessons learned thus far by developing and using the J3 Toolsuite:

- Creating a flexible system to aid the development of autonomic EJB applications is hard, e.g., not all applications want to monitor the same types of data sets. A DSML must therefore be flexible to incorporate unanticipated data sets, yet also handle the most common cases intuitively. Striking this balance between flexibility and general case utility took patience and iteration.

- Developing adaptations for an application is hard. Most developers do not think about designing components that can be adapted, swapped, restarted, or reconfigured to handle errors. Providing a DSML to aid developers in seeing the crosscutting adaptive concerns was hard.

- Creating a model of the mapping from components to QoS properties and adaptive behavior greatly enhances the ability of developers to understand the complex behavior of autonomic systems that would ordinarily be buried in hundreds of source files.

- Constraint checking and code generation can greatly reduce and/or eliminate hard-to-debug runtime errors, such as JNDI naming errors. In future work, we are developing increasingly sophisticated autonomic distributed applications using our J3 Toolsuite to serve as a testbed for investigating various autonomic architectures. We are also enhancing these tools to increase their expressive and code generation 
capabilities. Finally, we are planning to use our MDD tools to investigate developing applications for multi-core processors and optimizing the allocation of threads and components to cores.

The J3 Toolsuite DSMLs, tools, and frameworks are available in open-source form at www.sourceforge.net/projects/j2eeml.

\section{References}

1. Alur D, Crupi J, Malks D (2003) J2EE Core Patterns. Sun Microsystems Press

2. Asikainen T, Männistö T, Soininen T (2004) Representing Feature Models of Software Product Families Using a Configuration Ontology ECAI 2004. Workshop on Configuration 3. Candea G, Kawamoto S, Fujiki Y, Friedman G, Fox A (2004) Microreboot -- A Technique for Cheap Recovery. In: Proc. 6th Symposium on Operating Systems Design and Implementation (OSDI), San Francisco, CA, December

4. Candea G, Fox A (2001) Designing for High Availability and Measurability. In: Proc. of the 1st Workshop on Evaluating and Architecting System Dependability

5. Coulson G, Baichoo S, Moonian O (2002) A Retrospective on the Design of the GOPI Middleware Platform. In: ACM Multimedia Journal

6. Eymann T, Reinicke M (2003) Self-Organizing Resource Allocation for Autonomic Networks. In: Proc. DEXA Workshops

7. Gamma E, Helm R, Johnson R, Vlissides J (1995) Design Patterns: Elements of Reusable Object-Oriented Software. Addison-Wesley, New York

8. Gray J, Roychoudhury S (2004) A Technique for Constructing Aspect Weavers Using a Program Transformation Engine. In: Proc. of AOSD '04, Lancaster, UK

9. Hatcliff J, Deng W, Dwyer M, Jung G, Prasad V (2003) Cadena: An Integrated Development, Analysis, and Verification Environment for Component-based Systems. In: Proc. of the 25th International Conference on Software Engineering, Portland, OR

10. IBM, Autonomic Computing Toolkit,

www106.ibm.com/developerworks/autonomic/overview.html.

11. IBM Developerworks, Specification: Common Base Event.

(www106.ibm.com/developerworks/webservices/library/ws-cbe/).

12. Kang K, Cohen SG, Hess JA, Novak WE, Peterson.SA (1990) Feature Oriented Domain Analysis (FODA) - Feasibility Study. Technical report, Carnegie-Mellon University 
13. Kephart JO, Chess DM (2003) The Vision of Autonomic Computing. IEEE Computer, January

14. Ledeczi A, Bakay A, Maroti M, Volgysei P, Nordstrom G, Sprinkle J, Karsai G (2001) Composing Domain-Specific Design Environments. IEEE Computer, November

15. Ledeczi A (2001) The Generic Modeling Environment. In: Proc. Workshop on Intelligent Signal Processing, Budapest, Hungary

16. Loyall J, Bakken D, Schantz R, Zinky J, Karr D, Vanegas R (1998) QoS Aspect Languages and Their Runtime Integration. In: Proc. of the Fourth Workshop on Languages, Compilers and Runtime Systems for Scalable Components

17. Matena V, Hapner M (1999) Enterprise Java Beans Specification, Version 1.1. Sun Microsystems, December

18. Melcher B, Mitchell B (2004) Towards an Autonomic Framework: Self-Configuring Network Services and Developing Autonomic Applications. Intel Technology Journal, November

19. Oppenheimer D, Ganapathi A, Patterson D (2003) Why do Internet services fail, and what can be done about it?. In: Proc. USENIX Symposium on Internet Technologies and Systems, March

20. Tarr P, Ossher H, Harrison W, Sutton SM (1999) N Degrees of Separation: MultiDimensional Separation of Concerns. In: Proc. 21st International Conference on Software Engineering, May

21. Wang N, Schmidt DC, Gokhale A, Rodrigues C, Natarajan B, Loyall J, Schantz R., Gill C (2003) QoS-enabled Middleware. In Q Mahmoud (ed) Middleware for Communications. Wiley and Sons, New York, (2003)

22. White J, Schmidt D (2005) Simplifying the Development of Product-Line Customization Tools via MDD. In: Proc. Workshop: MDD for Software Product Lines, ACM/IEEE 8th International Conference on Model Driven Engineering Languages and Systems, October 23. Zinky J, Bakken D, Schantz. R (1997) Architectural Support for Quality of Service for CORBA Objects. In: Theory and Practice of Object Systems, Vol. 3, No. 1 